\title{
Research on the diagnostic value of serum tumor markers and CTC in the diagnosis of liver cancer.
}

\author{
Siqian Lu', Ying Luo ${ }^{2 *}$ \\ ${ }^{1}$ Faculty of Environmental Science and Engineering, Kunming University of Science and Technology, Kunming, \\ 650500, PR China \\ ${ }^{2}$ Faculty of Life Science and Technology and Faculty of Environmental Science and Engineering, Lab of Molecular \\ Genetics of Aging and Tumor, Kunming University of Science and Technology, Kunming, 650500, PR China
}

\begin{abstract}
In today's era, with a variety of different factors, cancer has become a common disease in today's times; the cancer is very different according to the different types of organizations. As one of the most common malignant tumors, the incidence and mortality of liver cancer is relatively high. In this study, based on the current situation of the incidence and mortality of liver cancer, the importance and accuracy of serum tumor markers and CTC in the diagnosis of liver cancer were analyzed. The results show that serum tumor markers and CTC can improve the accuracy of diagnosis.
\end{abstract}

Keywords: Serum tumor, Marker, CTC technique, Liver cancer diagnosis.

Accepted on May 05, 2017

\section{Introduction}

With the development of the times and the acceleration of the process of industrialization, people's living environment and things have begun to be more unsafe, so that some of the more serious diseases have continued to occur. Among them, the impact of cancer on people is extremely serious. And because of the influence of various factors, cancer may cause some harm to people's life and normal behavior, and it may cause some diseases that can't be recovered in some tissues, and even cause death.

Liver cancer is a malignant tumor which is serious to human beings; it is the main cause of human death of a neoplastic disease in the world. This kind of disease is a malignant tumor in the liver, with the gradual spread of the tumor, it may spread in a variety of tissues, so as to cause the damage of digestive system and cause the loss of liver tissue, at the same time, it can cause cancer cells in different tissues of the body, which may cause death and injury. As one of the most common malignant tumors in the current era, the tumor cells have the characteristics of high invasiveness and wide transfer range in the course of liver cancer, so that it is more difficult to prevent and treat it more effectively. Therefore, it is only through the study of the mechanism of the activation of the relevant genes of liver cancer cells to analyze the pathogenesis.

\section{State of the Art}

The pathogenesis of liver cancer and its main influencing factors

As a serious malignant tumor, liver cancer has caused some problems to the people of the world. The main part of the disease is liver tissue. People's unhealthy habits and poor eating habits may be one of the main causes of liver cancer. Because of the development trend of the industrialization process; the pollution of the air environment has further caused the malignant tumor of liver cancer. According to the forecast, due to the rapid development of industrialization in the present era, the deterioration of the environment and the air pollution make it possible to gradually increase the risk of liver cancer for people. Some researchers predict that in the next ten years, the number of deaths caused by liver cancer will gradually increase [1]. Therefore, it is necessary to develop tumor diagnosis and treatment methods, so as to make the control of liver cancer disease have a greater degree of improvement.

\section{The importance of prediction methods and treatment of liver cancer}

As a major cancer threat to human health and life safety, the pathogenesis of liver cancer is still not clearly understood, especially in the case of the increasing number of influencing factors of liver cancer and the gradual increase in mortality of liver cancer in the current era, the treatment and prediction of liver cancer has become one of the most important issues in the research of the disease [2]. Many researchers have begun to 
study the pathogenesis of liver cancer. Especially in today's era of rapid development of computer technology and biotechnology, a lot of technologies have begun to be applied in the diagnosis of liver cancer, such as the role of CTC technology and serum tumor markers in the diagnosis and treatment of liver cancer; the rapid development of these technologies for the diagnosis and treatment of liver cancer has made a certain role in promoting.

\section{Methodology}

\section{Study on the incidence of liver cancer in China}

Along with our country entering the industrialization era, our country's air environment has caused a certain degree of pollution, coupled with the poor state of life and the bad habits, the incidence of liver cancer in China is increasing year by year (Figure 1). In view of this phenomenon, our country has gradually started to predict and treat liver cancer as one of the important research topics in the development of medicine. Although there are a lot of funds into the diagnosis and treatment of liver cancer, the relevant diagnosis and treatment technology and theory is relatively backward. Therefore, the treatment and prediction of liver cancer is still not mature enough. This study analyzed the application status of serum tumor markers and CTC technology, and discussed the accuracy of which in the treatment of liver diseases.

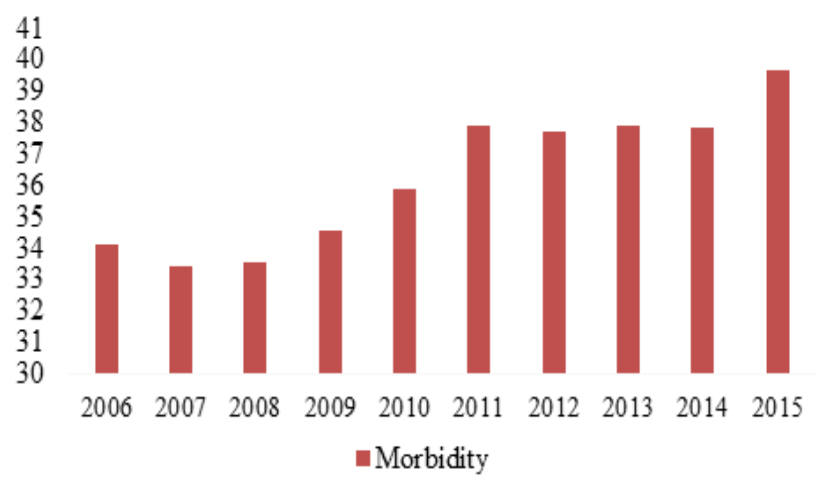

Figure 1. The incidence of liver cancer in China.

\section{Research methodology}

Firstly, through the relevant works and literature reading, the relevant theories were researched and analyzed, and the related theories of the serum tumor markers and CTC technology were further improved, thus, further research and discussion were made on the basis of the relative theories; Secondly, this study selected liver cancer patients as the objects of study, the control of patient's condition before and after the application of serum tumor markers and CTC technology was compared and analyzed, so that the practical value of serum tumor markers and CTC technology was determined from the point of view of examples, and the further analysis and discussion of its advantages were made.

\section{Result Analysis and Discussion}

\section{The application of serum tumor markers and CTC technology in liver cancer}

By summarizing and analyzing the relevant data, this study summarized the related theories of serum tumor markers and CTC technology, the specific chemical components in serum were combined with some target cells in liver cancer cells, if they could be effectively combined, the tumor cells were activated; if they could not be combined, so that there was no lesion, the technology was more accurate and systematic in the diagnosis of tumor cells.

\section{Analysis of the status of patient treatment before and after the application of serum tumor markers and CTC technology}

The investigation of all patients with liver cancer was carried out. The results are shown in Figure 2. It can be found that doctors can predict the tumor cells by the combination of markers in the serum tumor markers and CTC technology, so that patients with liver cancer can get more accurate treatment time and curative effect.

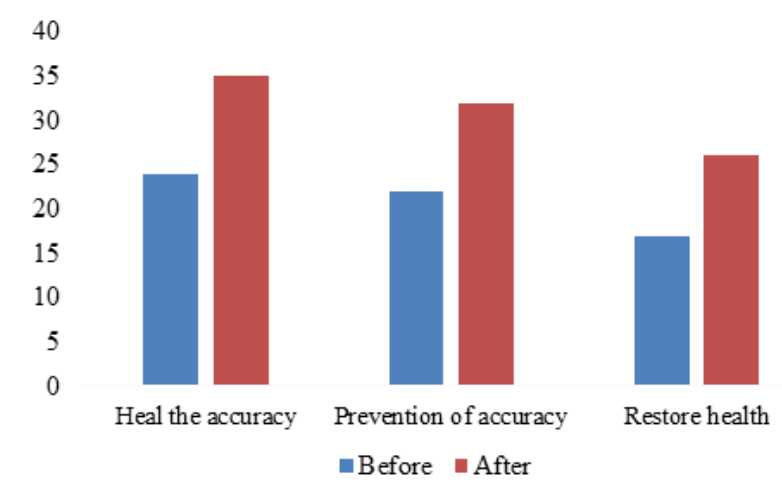

Figure 2. Analysis of the status of patient treatment before and after the application of serum tumor markers and CTC technology

\section{Conclusions}

Liver cancer is a kind of malignant tumor disease which has a great influence on people's health; the research on the mechanism of the pathogenesis of malignant tumor is relatively small, and it is still unable to obtain effective treatment. In this study, the related diagnosis and treatment technology of serum tumor markers and CTC were analyzed. The purpose of this study is to provide some theoretical basis and scientific support for the treatment and prevention of liver cancer.

\section{References}

1. Jemal A, Bray F, Center MM. Global cancer statistics. CA Cancer J Clin 2011; 61: 69-90.

2. Tripathi V, Ellis JD, Shen Z. The nuclear-retained noncoding RNA MALAT1 regulates alternative splicing by 
Research on the diagnostic value of serum tumor markers and CTC in the diagnosis of liver cancer

modulating SR splicing factor phosphorylation. Mol Cell 2010; 39: 925-938.

\section{*Correspondence to}

Ying Luo

Faculty of Life Science and Technology

Lab of Molecular Genetics of Aging and Tumor

Kunming University of Science and Technology

PR China 